\title{
Picky Eating in School-Aged Children: Sociodemographic Determinants and the Associations with Dietary Intake
}

\author{
Hebah Alawi Kutbi
}

check for updates

Citation: Kutbi, H.A. Picky Eating in School-Aged Children: Sociodemographic Determinants and the Associations with Dietary Intake. Nutrients 2021, 13, 2518. https:// doi.org/10.3390/nu13082518

Academic Editor: Cristina Padez

Received: 16 June 2021

Accepted: 21 July 2021

Published: 23 July 2021

Publisher's Note: MDPI stays neutral with regard to jurisdictional claims in published maps and institutional affiliations.

Copyright: (C) 2021 by the author. Licensee MDPI, Basel, Switzerland. This article is an open access article distributed under the terms and conditions of the Creative Commons Attribution (CC BY) license (https:/ / creativecommons.org/licenses/by/ $4.0 /)$.
Clinical Nutrition Department, Faculty of Applied Medical Sciences, King Abdulaziz University, P.O. Box 80215, Jeddah 21589, Saudi Arabia; hkutbi@kau.edu.sa

\begin{abstract}
Children exhibiting picky eating behavior often demonstrate strong food preferences and rejection of particular foods or food texture, which may lead to limited dietary variety and possibly inadequate or unhealthy diet. Yet, the relationship between picky eating and nutrient intake in school-aged children has not been established previously. This study aimed to investigate the sociodemographic determinants of picky eating and the associations between picky eating and dietary intake in children. Data of 424 healthy Saudi children aged 6-12 years were collected from their mothers. A child's picky eating habits were captured using a validated questionnaire. Sociodemographic characteristics of the children were assessed. Dietary data, including $24 \mathrm{~h}$ dietary recalls and frequency of fruit, vegetable, and milk consumption, were collected by dietetic professionals using phone-administered interviews. Compared to those of normal-weight mothers, children of mothers with obesity had higher odds of being in the highest tertile of picky eating (OR $=1.93 ; 95 \%$ CI 1.02, 3.63). Children exhibiting higher levels of picky eating consumed less fruits $(B=-0.03 ; 95 \% \mathrm{CI}-0.06$, $-0.01)$, vegetables $(B=-0.05 ; 95 \% C I-0.07,-0.02)$, and protein $(B=-0.21 ; 95 \% C I-0.33,-0.09)$, and had higher consumption of trans fatty acid intake $(B=1.10 ; 95 \%$ CI $0.06,2.15)$. Children with higher levels of picky eating presented unhealthy dietary behaviors. Future studies are needed to examine the long-term effect of picky eating on cardiovascular health. Dietary behaviors of mothers with obesity must be taken into consideration when designing intervention programs aiming to improve eating behaviors of children.
\end{abstract}

Keywords: picky eating; picky eaters; nutrient intake; dietary intake; children; cardiovascular health

\section{Introduction}

Picky eating is a common eating behavior among children [1-6], often causing considerable stress to parents and caregivers [6]. Children exhibiting picky eating behavior often demonstrate strong food preferences and rejection of particular foods or food texture [7], which may lead to limited dietary variety and possibly inadequate or unhealthy diet [8,9]. This is particularly relevant when the child rejects the consumption of healthy foods as it may raise the concern for not meeting dietary recommendations or replacing healthy foods by unhealthy food choices [8].

As parents become concerned for the child not eating a variety of foods, they may utilize different feeding practices to overcome the child's pickiness, which may further hinder the development of healthy dietary behaviors. For instance, the current data indicate that mothers of picky eaters tend to use more pressure on the children to eat and less monitoring of their dietary behaviors [10]. As such, parents may allow the child to consume any of the preferred foods to compensate for the limited food intake [10].

The existing evidence highlight the need to identify barriers to healthy food choices and a clear understanding of factors that impede acceptance and consumption of healthy foods [7]. Therefore, a substantial body of literature has examined the relationship between picky eating and food intake among children. A common theme is that picky eaters tend to demonstrate unhealthy dietary behaviors as compared to non-picky eaters; they have lower 
intake of vegetables and fruits $[3,8,10-12]$, meat, and fish $[10,12]$ than non-picky eaters, and higher intakes of savory snacks, sweets, sugary drinks, and French fries $[3,10,13]$. However, there is no clear evidence that nutrient intakes of picky eaters differ than that of non-picky eaters. Only one study quantified nutrient intakes of preschoolers and reported lower iron and zinc intakes in picky eaters [8]. Additionally, very little is known about health outcomes of picky eating [14]. Some studies investigated the associations of picky eating with children's growth and weight status and reported inconsistent findings [15,16]. Other studies suggest that picky eaters experience constipation more commonly than non-picky eaters [17,18].

Existing data suggests high prevalence of picky eating among children. For instance, a study conducted among Chinese children reported a prevalence of 59.3\% [1]. In Saudi Arabia, the prevalence of picky eaters was estimated as $89.8 \%$ [2]. Other studies reported rates of picky eaters ranging from 13 to $47.4 \%$ [3-6]. As such, many studies have been devoted to understanding the factors associated with picky eating. Findings indicated that preschool-aged children of low-income households are more vulnerable to picky eating compared to those of high-income households [10]. The current evidence also suggests that parent-child interaction and the child's sensory sensitivity are associated with the development of picky eating [19]. Children with greater sensory sensitivity are more prone to become picky eaters. Parental sensitivity and the lower parental structuring may also increase the likelihood of children exhibiting picky eating, which could be due to the limited opportunity for teaching and learning about foods [19].

Due to the high prevalence of picky eating among children and the limited availability of studies that investigate the relationship between picky eating and nutrient intake of children, the present study aimed to explore the sociodemographic determinants of picky eating and to investigate the associations between picky eating and dietary intake in school-aged children.

\section{Materials and Methods}

\subsection{Study Sample}

For this cross-sectional study, a minimum of 346 children was required, determined by an expected correlation coefficient of 0.15 , power of $80 \%$, and $\alpha=0.05$ [20]. As mothers in many Arab countries are considered responsible for child feeding [21], data on the children were collected from their mothers. A questionnaire link was circulated using social media (WhatsApp, Twitter, Instagram, and Telegram) along with a statement describing the study objectives and protocol, confidentiality statement, and consent for participation. Mothers of multiple children were instructed to provide data of the youngest child. The questionnaire included questions on sociodemographic characteristics and eating behaviors of the child and asked for a convenient date and time to conduct a telephone interview for $24 \mathrm{~h}$ dietary recall(s) collection.

Five hundred and thirty-nine mothers completed the online questionnaire. Data of healthy Saudi children (6-12 years old) residing in Saudi Arabia were included. The exclusion criteria included participants who did not report dietary data $(14.7 \%, n=79)$ and children with chronic illnesses or food allergies $(6.68 \%, n=36)$. A total of 424 children were included in the sample. This study was approved by the Research and Ethics Committee, Faculty of Applied Medical Science at King Abdulaziz University (FAMS-EC-2020-0010).

\subsection{Measures}

\subsubsection{Sociodemographic Characteristics}

Data were collected regarding the child's sex and age, region of residence, birth order (first child vs. not), breastfeeding initiation, monthly household income, paternal and maternal education level, maternal age and employment status, and whether the father is involved in child feeding and lives with the child. Mothers were also requested to report their current weight and height, and the body mass index (BMI) was calculated. Maternal weight status was determined based on the World Health Organization (WHO) criteria; maternal weight was defined as normal if the BMI was $18.5-24.9 \mathrm{~kg} / \mathrm{m}^{2}$, underweight if the BMI was $<18.5 \mathrm{~kg} / \mathrm{m}^{2}$, and overweight or obese if the BMI was $25.0-29.9$ or $\geq 30.0 \mathrm{~kg} / \mathrm{m}^{2}$, respectively [22]. 


\subsubsection{Picky Eating Scale}

The degree of picky eating was assessed using the picky eating subscale from the Child Eating Behavior Questionnaire [23], which has been previously validated among children in Saudi Arabia [24]. The picky eating construct consists of three items: (1) "My child is fussy or picky about what he/she eats"; (2) "My child's diet consists of only a few foods"; and (3) "My child is unwilling to eat many of the foods that our family eats at mealtime." Response options for each item ranged from 1 "disagree" to 5 "agree." The average score of the three items was calculated, wherein a score of 5 indicated the highest level of picky eating. The internal reliability of the construct assessed by Cronbach's alpha was acceptable $(\alpha=0.70)$. To evaluate the associations of child characteristics with the degree of picky eating, children were divided into tertiles based on their average picky eating scale score.

\subsubsection{Dietary Assessment}

Dietary data were collected by trained dietetic professionals, and two registered dietitians were responsible for overseeing the collection of dietary data, field validation, and data entry. A phone interview reminder was sent to each mother as a text message a day earlier. During the interview, mothers were requested to estimate the frequency and quantity of the child's consumption of fruits, vegetables, and milk. The frequency of consumption was reported as "per day," "per month," or "per week," and the average frequency was calculated to represent children's intake per day. Additionally, a comprehensive education was provided on the serving size of foods and data to disclose when reporting the $24 \mathrm{~h}$ dietary recall(s), such as the number of servings, food description, the name of ready-to-eat food manufacturers, etc. Pictures of standardized measurements were sent to mothers to further assist in estimating portion size of food consumed and to ensure correct estimations of food quantity. Another phone interview was scheduled to collect $24 \mathrm{~h}$ dietary recall, and mothers were requested to have the child and whoever assists in feeding the child participate in the phone interview. To adjust for within individual variations, three inconsecutive $24 \mathrm{~h}$ dietary recalls (one weekend day and two weekdays) were obtained for 168 randomly selected children (39.6\%) within two weeks from the first $24 \mathrm{~h}$ dietary recall.

The dietary data were analyzed using the dietary analysis software Nutritics, research edition (version 5.6, Dublin, Ireland). The Nutritics software includes official national databases for countries such as Saudi Arabia, and a database of universal Arabic foods. Local standardized food recipes were entered into the software. Density of macronutrients were then calculated as percentages of energy intake, and density of each micronutrient was calculated by dividing the nutrient intake per unit by $1000 \mathrm{kcal}$.

\subsection{Statistical Analysis}

Data were illustrated as median (interquartile range), mean \pm standard deviation, and percentages (frequency). Chi-square test was used to assess differences in child characteristics across the three tertiles of picky eating, whereas Kruskal-Wallis test was used to investigate differences in dietary intakes (2-sided tests). Multinomial regression adjusted for child sex and age was used to examine the independent associations between child characteristics and tertiles of picky eating, wherein tertile I was set as the reference category. The associations between picky eating and dietary intakes were further investigated using linear regression analyses adjusted for child sex and age as covariates. The regression analyses were performed at $95 \%$ confidence level. The Statistical Package for Social Sciences (SPSS) was used for data analyses.

\section{Results}

Nearly half of the children were boys $(49.5 \%, n=210)$ and lived in households with annual income above 10,000 Saudi riyals (SRs) $(54.0 \%, n=229)$. Over two-thirds of mothers $(75.2 \%, n=319)$ and fathers $(63.2 \%, n=268)$ had a college degree or higher. The majority of 
children in our sample were living with their mothers and fathers $(89.4 \%, n=379)$, and about half of the fathers were reported to be participating in child feeding $(47.4 \%, n=201)$. Thirty-eight percent of the mothers had normal bodyweight $(n=161)$. The prevalence of underweight, overweight, and obesity among mothers was $3.10 \%(n=13), 36.8 \%(n=156)$, and $22.2 \%(n=94)$, respectively.

The median picky eating scale score across the total study sample was 3.00 out of a total score of $5.00(2.33-4.00)$, with a mean score of $3.07 \pm 1.07$. Median picky eating scale score among children in tertile I was 2.00 (1.67-2.33), with a mean score of $1.89 \pm 0.46$. Median and mean picky eating scale scores for children in tertiles II and III were 3.00 (2.67-3.33), $3.00 \pm 0.25$ and 4.33 (3.67-4.67), $4.25 \pm 0.47$, respectively. Table 1 describes children's characteristics stratified by tertiles of picky eating. The univariate analyses did not indicate any significant difference in child characteristics across tertiles of picky eating $(p>0.05)$. However, the multinomial regression analyses adjusted for child sex and age suggests that compared to children of normal-weight mothers, children of mothers with obesity have higher odds of being in the highest tertile of picky eating ( $\mathrm{OR}=1.93 ; 95 \% \mathrm{CI}$ $1.02,3.63$ ) (Table 2).

Table 1. Differences in characteristics of children among tertiles of picky eating $(n=424)^{\text {a }}$.

\begin{tabular}{|c|c|c|c|c|c|}
\hline \multirow{2}{*}{\multicolumn{2}{|c|}{ Characteristic }} & \multicolumn{3}{|c|}{ Picky Eating } & \multirow[b]{2}{*}{$p$-Value } \\
\hline & & \multirow[t]{2}{*}{$\begin{array}{r}\text { Tertile I } \\
(n=145)\end{array}$} & \multirow{2}{*}{$\begin{array}{c}\begin{array}{l}\text { Tertile II } \\
(n=126)\end{array} \\
41(32.5 \%)\end{array}$} & \multirow{2}{*}{$\begin{array}{c}\begin{array}{c}\text { Tertile III } \\
(n=153)\end{array} \\
56(36.6 \%)\end{array}$} & \\
\hline \multirow{3}{*}{ Age in years, $n(\%)$} & $6-7$ & & & & \multirow{3}{*}{0.88} \\
\hline & $8-9$ & $44(30.3 \%)$ & $36(28.6 \%)$ & $46(30.1 \%)$ & \\
\hline & $10-12$ & $54(37.2 \%)$ & $49(38.9 \%)$ & $51(33.3 \%)$ & \\
\hline \multirow{2}{*}{ Sex, $n(\%)$} & Boys & $67(46.2 \%)$ & $56(44.4 \%)$ & $87(56.9 \%)$ & \multirow{2}{*}{0.07} \\
\hline & Girls & $78(53.8 \%)$ & $70(55.6 \%)$ & $66(43.1 \%)$ & \\
\hline \multirow{5}{*}{ Region of residence, $n(\%)$} & Western & $80(55.2 \%)$ & $73(57.9 \%)$ & $89(58.2 \%)$ & \multirow{5}{*}{0.78} \\
\hline & Central & $18(12.4 \%)$ & $13(10.3 \%)$ & $25(16.3 \%)$ & \\
\hline & Eastern & $20(13.8 \%)$ & $19(15.1 \%)$ & $14(9.2 \%)$ & \\
\hline & Southern & $17(11.7 \%)$ & $14(11.1 \%)$ & $17(11.1 \%)$ & \\
\hline & Northern & $10(6.9 \%)$ & $7(5.6 \%)$ & $8(5.2 \%)$ & \\
\hline \multirow{2}{*}{ Birth order, $n(\%)$} & Not first child & $89(61.4)$ & $84(66.7)$ & $90(58.8)$ & \multirow[b]{2}{*}{0.34} \\
\hline & First child & $56(38.6)$ & $42(33.3)$ & $63(41.2)$ & \\
\hline \multirow{3}{*}{ Maternal age in years, $n(\%)$} & $<31$ & $30(20.7 \%)$ & $19(15.1 \%)$ & $27(17.6 \%)$ & \multirow{3}{*}{0.59} \\
\hline & $31-40$ & $82(56.6 \%)$ & $69(54.8 \%)$ & $88(57.5 \%)$ & \\
\hline & $>40$ & $33(22.8 \%)$ & $38(30.2 \%)$ & $38(24.8 \%)$ & \\
\hline \multirow{4}{*}{ Maternal weight status, $n(\%)$} & Normal weight & $58(40.0)$ & $49(38.9)$ & $54(35.3)$ & \multirow{4}{*}{0.49} \\
\hline & Underweight & $6(4.10)$ & $4(3.20)$ & $3(2.00)$ & \\
\hline & Overweight & $56(38.6)$ & $46(36.5)$ & $54(35.3)$ & \\
\hline & Obese & $25(17.2)$ & $27(21.4)$ & $42(27.5)$ & \\
\hline \multirow{2}{*}{ Maternal education, $n(\%)$} & $\leq$ High school/diploma & $43(29.7)$ & $25(19.8)$ & $37(24.2)$ & \multirow{2}{*}{0.17} \\
\hline & $\geq$ College degree & $102(70.3)$ & $101(80.2)$ & $116(75.8)$ & \\
\hline \multirow{2}{*}{ Paternal education, $n(\%)$} & $\leq$ High school/diploma & $50(34.5)$ & $48(38.1)$ & $58(37.9)$ & \multirow{2}{*}{0.78} \\
\hline & $\geq$ College degree & $95(65.5)$ & $78(61.9)$ & $95(62.1)$ & \\
\hline \multirow{2}{*}{$\begin{array}{c}\text { Maternal employment status, } \\
n(\%)\end{array}$} & Unemployed & $85(58.6 \%)$ & $68(54 \%)$ & $98(64.1 \%)$ & \multirow{2}{*}{0.23} \\
\hline & Employed & $60(41.4 \%)$ & $58(46 \%)$ & $55(35.9 \%)$ & \\
\hline \multirow{2}{*}{$\begin{array}{l}\text { Any breastfeeding during first } \\
6 \text { months of life, } n(\%)\end{array}$} & No & $19(13.1)$ & $13(10.3)$ & $23(15.0)$ & \multirow[b]{2}{*}{0.51} \\
\hline & Yes & $126(86.9)$ & $113(89.7)$ & $130(85.0)$ & \\
\hline \multirow{2}{*}{ Father lives with the child, $n(\%)$} & No & $9(6.20)$ & $16(12.7)$ & $20(13.1)$ & \\
\hline & Yes & $136(93.8)$ & $110(87.3)$ & $133(86.9)$ & 0.10 \\
\hline & No & $30(20.7)$ & $26(20.6)$ & $42(27.5)$ & \\
\hline $\begin{array}{l}\text { Paternal involvement in child } \\
\text { feeding } n(\%)\end{array}$ & Sometimes & $39(26.9)$ & $35(27.8)$ & $51(33.3)$ & 0.16 \\
\hline & Yes & $76(52.4)$ & $65(51.6)$ & $60(39.2)$ & \\
\hline & $<4000$ SR & $10(6.9 \%)$ & $9(7.1 \%)$ & $10(6.5 \%)$ & \\
\hline & $4000-6000$ SR & $20(13.8 \%)$ & $19(15.1 \%)$ & $23(15 \%)$ & \\
\hline Monthly household income, $n(\%)$ & $6001-10,000$ SR & $35(24.1 \%)$ & $23(18.3 \%)$ & $46(30.1 \%)$ & 0.40 \\
\hline & $10,001-15,000 \mathrm{SR}$ & $39(26.9 \%)$ & $30(23.8 \%)$ & $39(25.5 \%)$ & \\
\hline & $>15,000 \mathrm{SR}$ & $41(28.3 \%)$ & $45(35.7 \%)$ & $35(22.9 \%)$ & \\
\hline
\end{tabular}

${ }^{a}$ Chi-square test was used for categorical variables. Abbreviation: SR, Saudi riyal. 
Table 2. Adjusted odds ratio for the association between child characteristics and picky eater status ${ }^{\text {a }}$.

\begin{tabular}{|c|c|c|c|c|}
\hline \multirow[b]{2}{*}{ Predictor } & \multicolumn{4}{|c|}{ Adjusted Odds Ratio and 95\% Confidence Intervals } \\
\hline & $\begin{array}{l}\text { Tertile II to Reference } \\
\text { Category }\end{array}$ & $p$-Value & $\begin{array}{l}\text { Tertile III to Reference } \\
\text { Category }\end{array}$ & $p$-Value \\
\hline \multicolumn{5}{|c|}{ Sex } \\
\hline Boy & $0.93(0.57,1.50)$ & 0.76 & $1.52(0.96,2.41)$ & 0.07 \\
\hline Girl & \multicolumn{4}{|c|}{1.00 (Reference category) } \\
\hline \multicolumn{5}{|c|}{ Region of residence } \\
\hline Western & $1.30(0.47,3.64)$ & 0.62 & $1.45(0.54,3.90)$ & 0.47 \\
\hline Central & $1.04(0.31,3.46)$ & 0.95 & $1.82(0.59,5.58)$ & 0.30 \\
\hline Eastern & $1.38(0.43,4.37)$ & 0.59 & $0.86(0.27,2.76)$ & 0.80 \\
\hline Southern & $1.18(0.35,3.97)$ & 0.79 & $1.27(0.39,4.08)$ & 0.69 \\
\hline Northern & \multicolumn{4}{|c|}{1.00 (Reference category) } \\
\hline \multicolumn{5}{|c|}{ Age } \\
\hline $6-7$ years old & $0.97(0.55,1.71)$ & 0.90 & $1.23(0.71,2.13)$ & 0.45 \\
\hline 8-9 & $0.90(0.50,1.62)$ & 0.72 & $1.13(0.64,1.99)$ & 0.67 \\
\hline $10-12$ & \multicolumn{4}{|c|}{1.00 (Reference category) } \\
\hline \multicolumn{5}{|l|}{ Birth order } \\
\hline Not first child & $1.26(0.76,2.10)$ & 0.38 & $0.92(0.57,1.49)$ & 0.74 \\
\hline First child & \multicolumn{4}{|c|}{1.00 (Reference category) } \\
\hline \multicolumn{5}{|c|}{ Any breastfeeding during first 6 months of life } \\
\hline No & $0.76(0.36,1.61)$ & 0.47 & $1.18(0.61,2.28)$ & 0.63 \\
\hline Yes & \multicolumn{4}{|c|}{1.00 (Reference category) } \\
\hline \multicolumn{5}{|c|}{ Maternal education } \\
\hline$\leq$ High school/diploma & $0.58(0.33,1.02)$ & 0.06 & $0.79(0.47,1.33)$ & 0.38 \\
\hline$\geq$ College degree & \multicolumn{4}{|c|}{1.00 (Reference category) } \\
\hline \multicolumn{5}{|c|}{ Paternal education } \\
\hline$\leq$ High school/diploma & $1.17(0.71,1.92)$ & 0.54 & $1.14(0.71,1.83)$ & 0.59 \\
\hline$\geq$ College degree & \multicolumn{4}{|c|}{1.00 (Reference category) } \\
\hline \multicolumn{5}{|c|}{ Maternal employment status } \\
\hline Unemployed & $0.82(0.51,1.33)$ & 0.43 & $1.28(0.80,2.05)$ & 0.30 \\
\hline Employed & \multicolumn{4}{|c|}{1.00 (Reference category) } \\
\hline \multicolumn{5}{|c|}{ Household income } \\
\hline$<4000$ & $0.82(0.30,2.21)$ & 0.69 & $1.15(0.43,3.10)$ & 0.78 \\
\hline $4000-6000$ & $0.86(0.40,1.84)$ & 0.69 & $1.35(0.63,2.88)$ & 0.44 \\
\hline $6001-10,000$ & $0.60(0.30,1.17)$ & 0.14 & $1.56(0.83,2.94)$ & 0.17 \\
\hline $10,001-15,000$ & $0.70(0.37,1.32)$ & 0.27 & $1.21(0.64,2.29)$ & 0.56 \\
\hline$>15,000$ & \multicolumn{4}{|c|}{1.00 (Reference category) } \\
\hline \multicolumn{5}{|c|}{ Maternal weight status } \\
\hline Underweight & $0.79(0.21,2.97)$ & 0.73 & $0.55(0.13,2.33)$ & 0.42 \\
\hline Overweight & $0.96(0.56,1.67)$ & 0.89 & $1.08(0.63,1.85)$ & 0.77 \\
\hline Obese & $1.27(0.65,2.49)$ & 0.49 & $1.93(1.02,3.63)$ * & 0.04 \\
\hline Normal & \multicolumn{4}{|c|}{1.00 (Reference category) } \\
\hline \multicolumn{5}{|c|}{ Father lives with the child } \\
\hline No & $0.16(0.02,1.40)$ & 0.10 & $0.10(0.01,0.76)$ * & 0.03 \\
\hline Yes & & 1.00 (Refer & ce category) & \\
\hline & Paternal involven & nt in child & ding & \\
\hline No & $1.02(0.55,1.91)$ & 0.94 & $1.75(0.98,3.14)$ & 0.06 \\
\hline Sometimes & $1.06(0.60,1.87)$ & 0.84 & $1.66(0.97,2.85)$ & 0.07 \\
\hline Yes & & 1.00 (Refer & ce category) & \\
\hline
\end{tabular}

a Models are adjusted for child age and sex. ${ }^{*} p<0.05$.

Dietary intakes of children stratified by tertiles of picky eating are shown in Table 3. Significant differences in the frequency of fruit and vegetable consumptions were observed across the picky eater tertile groups; Children in the lowest tertile of picky eating had a mean intake of $1.06 \pm 0.95$ servings of fruits per day, whereas children in the second and third tertiles consumed $1.04 \pm 0.87$ and $0.83 \pm 0.78$ serving of fruits per day, respectively $(p=0.01)$. Children in the lowest tertile, second tertile, and third tertile of picky eating consumed $1.04 \pm 0.79,0.96 \pm 0.82$, and $0.73 \pm 0.81$ serving of vegetables per day, respectively $(p<0.001)$. Furthermore, the mean intake of protein was found to be significantly different across the picky eater tertile groups; the mean protein intakes of children in first, second, 
and third tertiles were $15.9 \pm 4.03,14.9 \pm 3.92$, and $14.5 \pm 4.05 \mathrm{~g}$ per $1000 \mathrm{kcal}$ per day, respectively $(p<0.01)$.

Table 3. Dietary intakes of children stratified by tertiles of picky eating.

\begin{tabular}{|c|c|c|c|c|}
\hline \multirow[b]{2}{*}{ Dietary Intake a } & \multicolumn{3}{|c|}{ Picky Eating } & \multirow[b]{2}{*}{$p$-Value } \\
\hline & $\begin{array}{l}\text { Tertile I } \\
(n=145)\end{array}$ & $\begin{array}{l}\text { Tertile II } \\
(n=126)\end{array}$ & $\begin{array}{l}\text { Tertile III } \\
(n=153)\end{array}$ & \\
\hline Servings of milk per day & $\begin{array}{c}1.23 \pm 0.93 \\
1.00(0.43-2.50)\end{array}$ & $\begin{array}{c}1.26 \pm 0.99 \\
1.00(0.43-2.50)\end{array}$ & $\begin{array}{c}1.14 \pm 0.84 \\
1.00(0.43-1.00)\end{array}$ & 0.64 \\
\hline Servings of fruits per day & $\begin{array}{c}1.06 \pm 0.95 \\
1.00(0.43-1.00)\end{array}$ & $\begin{array}{c}1.04 \pm 0.87 \\
1.00(0.43-1.00)\end{array}$ & $\begin{array}{c}0.83 \pm 0.78 \\
1.00(0.43-1.00)\end{array}$ & $0.01 *$ \\
\hline Servings of vegetables per day & $\begin{array}{c}1.04 \pm 0.79 \\
1.00(0.43-1.00)\end{array}$ & $\begin{array}{c}0.96 \pm 0.82 \\
1.00(0.43-1.00)\end{array}$ & $\begin{array}{c}0.73 \pm 0.81 \\
0.43(0.14-1.00)\end{array}$ & $<0.01$ * \\
\hline Energy (kcal) & $\begin{array}{c}1314 \pm 385 \\
1205(1051-1561)\end{array}$ & $\begin{array}{c}1313 \pm 320 \\
1268(1101-1460)\end{array}$ & $\begin{array}{c}1310 \pm 335 \\
1266(1094-1467)\end{array}$ & 0.87 \\
\hline Carbohydrate (\%) & $\begin{array}{c}48.8 \pm 7.50 \\
48.7(44.0-52.9)\end{array}$ & $\begin{array}{c}50.3 \pm 6.79 \\
50.6(47.0-54.4)\end{array}$ & $\begin{array}{c}49.6 \pm 8.00 \\
49.3(44.7-54.2)\end{array}$ & 0.09 \\
\hline Fat $(\%)$ & $\begin{array}{c}33.3 \pm 6.35 \\
34.1(29.7-37.6)\end{array}$ & $\begin{array}{c}32.8 \pm 5.98 \\
32.5(28.3-36.0)\end{array}$ & $\begin{array}{c}33.9 \pm 7.30 \\
34.0(28.1-38.6)\end{array}$ & 0.28 \\
\hline Protein $(\%)$ & $\begin{array}{c}16.0 \pm 4.03 \\
15.7(13.2-18.3)\end{array}$ & $\begin{array}{c}14.9 \pm 3.92 \\
14.4(12.00-17.1)\end{array}$ & $\begin{array}{c}14.5 \pm 4.05 \\
14.1(11.7-17.0)\end{array}$ & $<0.01$ * \\
\hline Sugar (g/1000 kcal) & $\begin{array}{c}96.2 \pm 72.6 \\
71.6(47.6-124)\end{array}$ & $\begin{array}{c}96.4 \pm 53.2 \\
87.3(59.1-128)\end{array}$ & $\begin{array}{c}94.3 \pm 57.2 \\
82.5(51.8-126)\end{array}$ & 0.45 \\
\hline Fiber (g/1000 kcal) & $\begin{array}{c}14.6 \pm 9.70 \\
11.8(8.13-18.8)\end{array}$ & $\begin{array}{c}14.7 \pm 9.28 \\
12.7(8.31-19.3)\end{array}$ & $\begin{array}{c}13.8 \pm 9.79 \\
11.5(8.07-16.0)\end{array}$ & 0.64 \\
\hline Trans fat (g/1000 kcal) & $\begin{array}{c}0.50 \pm 0.65 \\
0.28(0.07-0.69)\end{array}$ & $\begin{array}{c}1.00 \pm 5.82 \\
0.29(0.07-0.65)\end{array}$ & $\begin{array}{c}6.56 \pm 85.4 \\
0.23(0.06-0.74)\end{array}$ & 0.76 \\
\hline Cholesterol (mg/1000 kcal) & $\begin{array}{c}296 \pm 236 \\
247(125-388)\end{array}$ & $\begin{array}{c}235 \pm 167 \\
192(104-323)\end{array}$ & $\begin{array}{c}260 \pm 234 \\
205(102-325)\end{array}$ & 0.07 \\
\hline Vitamin D (ug/1000 kcal) & $\begin{array}{c}34.8 \pm 99.4 \\
5.00(2.08-21.7)\end{array}$ & $\begin{array}{c}23.5 \pm 43.9 \\
4.74(2.00-13.7)\end{array}$ & $\begin{array}{c}25.0 \pm 42.2 \\
5.25(2.17-28.5)\end{array}$ & 0.87 \\
\hline Calcium (mg/1000 kcal) & $\begin{array}{c}818 \pm 592 \\
661(444-1022)\end{array}$ & $\begin{array}{c}798 \pm 463 \\
708(444-1047)\end{array}$ & $\begin{array}{c}779 \pm 444 \\
699(452-969)\end{array}$ & 0.92 \\
\hline Zinc (mg/1000 kcal) & $\begin{array}{c}6.85 \pm 4.27 \\
5.81(3.86-8.72)\end{array}$ & $\begin{array}{c}7.07 \pm 4.24 \\
5.92(4.32-8.72)\end{array}$ & $\begin{array}{c}6.55 \pm 4.25 \\
5.42(3.72-8.09)\end{array}$ & 0.42 \\
\hline Iron (mg/1000 kcal) & $\begin{array}{c}13.4 \pm 9.41 \\
10.7(7.07-16.7)\end{array}$ & $\begin{array}{c}12.8 \pm 7.26 \\
11.2(8.19-15.4)\end{array}$ & $\begin{array}{c}12.2 \pm 7.16 \\
10.7(7.62-14.3)\end{array}$ & 0.71 \\
\hline
\end{tabular}

${ }^{a}$ Dietary data are presented as mean \pm standard deviation and median (interquartile range). ${ }^{*} p<0.05$.

Multiple linear regression analyses adjusted for children's age and sex were conducted to examine the associations of picky eating with dietary intake of children (Table 4). Findings indicated inverse associations between picky eating scale scores and daily intakes of fruit $(B=-0.03 ; 95 \% C I-0.06$ to -0.01$)$, vegetable $(B=-0.05 ; 95 \% C I-0.07$ to -0.02$)$, and protein $(\mathrm{B}=-0.21 ; 95 \% \mathrm{CI}-0.33$ to -0.09$)$ and a positive association with trans fatty acid intake (B $=1.10 ; 95 \%$ CI 0.06 to 2.15$)$.

Table 4. Linear regression analyses of picky eating scale scores on dietary intakes of children a

\begin{tabular}{|c|c|c|c|c|c|}
\hline Dietary Intake & $\mathbf{R}^{2}$ & B & $\begin{array}{l}\text { Standard } \\
\text { Error }\end{array}$ & $\begin{array}{c}95 \% \text { Confidence } \\
\text { Intervals }\end{array}$ & $p$-Value \\
\hline Servings of milk per day & 0.01 & -0.03 & 0.01 & $-0.05,0.00$ & 0.06 \\
\hline Servings of fruits per day & 0.02 & -0.03 & 0.01 & $-0.06,-0.01$ & $0.01 *$ \\
\hline Servings of vegetables per day & 0.03 & -0.05 & 0.01 & $-0.07,-0.02$ & $<0.01$ * \\
\hline Energy (kcal) & 0.01 & -2.11 & 5.27 & $-12.4,8.24$ & 0.69 \\
\hline Carbohydrate (\%) & 0.01 & 0.09 & 0.11 & $-0.14,0.31$ & 0.44 \\
\hline Fat $(\%)$ & 0.01 & 0.11 & 0.10 & $-0.09,0.30$ & 0.29 \\
\hline Protein $(\%)$ & 0.03 & -0.21 & 0.06 & $-0.33,-0.09$ & $<0.01$ * \\
\hline Sugar (g/1000 kcal) & 0.003 & -0.51 & 0.94 & $-2.36,1.33$ & 0.59 \\
\hline Fiber (g/1000 kcal) & 0.01 & -0.19 & 0.15 & $-0.47,0.10$ & 0.21 \\
\hline Trans fat (g/1000 kcal) & 0.02 & 1.10 & 0.53 & $0.06,2.15$ & 0.04 * \\
\hline Cholesterol (mg/1000 kcal) & 0.02 & -6.08 & 3.28 & $-12.5,0.37$ & 0.07 \\
\hline
\end{tabular}


Table 4. Cont.

\begin{tabular}{|c|c|c|c|c|c|}
\hline Dietary Intake & $\mathbf{R}^{2}$ & B & $\begin{array}{l}\text { Standard } \\
\text { Error }\end{array}$ & $\begin{array}{l}\text { 95\% Confidence } \\
\text { Intervals }\end{array}$ & $p$-Value \\
\hline Vitamin D (ug/1000 kcal) & 0.02 & -0.80 & 1.02 & $-2.81,1.22$ & 0.44 \\
\hline Calcium (mg/1000 kcal) & 0.01 & -4.08 & 7.65 & $-19.1,11.0$ & 0.60 \\
\hline Zinc (mg/1000 kcal) & 0.02 & -0.07 & 0.06 & $-0.19,0.06$ & 0.31 \\
\hline Iron (mg/1000 kcal) & 0.02 & -0.18 & 0.12 & $-0.41,0.06$ & 0.15 \\
\hline
\end{tabular}

${ }^{\mathrm{a}}$ Models are adjusted for sex and age of children. ${ }^{*} p<0.05$.

\section{Discussion}

Findings of the present study showed that compared to those children of normalweight mothers, children of mothers with obesity have higher odds of being in the highest tertile of picky eating. Children exhibiting higher levels of picky eating consumed significantly fewer fruits and vegetables and less protein than those with lower levels, and higher amount of trans fat.

Dietary behaviors of children can be largely influenced by parental dietary behaviors and practices [15]. Children of parents who are picky eaters themselves tend to exhibit characteristics of picky eating behaviors [25]. Several studies have also observed parent-child commonalities in intakes of healthy and unhealthy foods, particularly for mothers [26-28]. In the present study, children of mothers with obesity were found to have higher odds of being in the highest tertile of picky eating than those of normal-weight mothers. This finding indicates that these children are more likely to experience a characteristic that is unique for mothers with obesity. A randomized controlled trial has demonstrated a significant association between obesogenic behaviors of children and mothers; children with obesity were more likely to meet behavioral recommendations for fast food intake, consumption of sugary drinks, and television viewing if their mothers presented lower levels of these behaviors [29]. Although picky eating in mothers has not been evaluated in this study, it is possible that the obese mothers themselves are experiencing picky eating, or that eating behaviors of the obese mothers are linked to their children's food pickiness. This could be particularly relevant as some studies have shown higher prevalence of obesity among picky eaters than non-picky eaters [25], although other studies reported inconsistent findings [30]. Further studies are needed to reveal how maternal obesity is linked to child's picky eating. When designing intervention programs aiming to improve eating behaviors of children, children of mothers with obesity must have a particular attention, and maternal dietary behaviors must be considered in order to achieve the desired outcomes.

Results of this study are aligned with previously reported findings in which children exhibiting picky eating behaviors tend to consume fewer fruits and vegetables and less protein than non-picky eaters [9]. Multiple factors could contribute to limited intake of these foods among children, such as food insecurity [31,32]. In the context of picky eating, some children might be more sensitive to tough texture than non-picky eaters [2], which could explain the limited intake of fruits, vegetables, and meat among children with higher levels of picky eating compared to those with the lower levels. Another possible explanation for the limited intake of protein-rich food sources is that picky eaters might be sensitive to the strong smell of these foods. In a previous study, children with higher levels of picky eating presented higher degrees of disgust and sensory sensitivity to food texture than non-picky eaters [16].

Our data indicated that children with higher levels of picky eating tend to consume greater amounts of trans fat. The higher consumption of trans fatty acids might be an indication for high intakes of fried or fast foods, baked goods such as cakes, cookies, crackers, and/or margarines [33,34]. Trans fatty acids are a form of unsaturated fat that can be presented naturally in dairy and meat or formed during hydrogenation of oils to enhance taste and texture of the foods [33,34]. A high consumption of trans fat raises the concern for children's cardiovascular health and lipid profile, particularly if this behavior was combined with fewer fruit and vegetable intakes. Existing evidence indicates that a high 
trans fatty acids intake is associated with lower high-density lipoprotein (HDL) and higher low-density lipoprotein (LDL) cholesterol levels, and increases the risk of developing heart diseases and type 2 diabetes [35]. The American Heart Association recommends limiting intakes of trans fatty acid and sugar while increasing fruit and vegetable consumption in order to prevent the development of cardiovascular disease in growing children [36]. As such, food choices of picky eaters must be carefully monitored.

Parents of picky eating children worry the most when the child rejects healthy foods and they may, therefore, pressure the child to eat the rejected food, although this feeding practice has been found to be counterproductive $[37,38]$. Alternatively, children may benefit from being surrounded by healthy food environment, as recent evidence indicates a negative association between healthy home food environment and picky eating in children [37].

The present study is among the first to investigate the association of picky eating with nutrient intake of school-aged children. Picky eating was assessed using a previously validated measurement tool that showed high internal reliability among the sample. However, this study is limited by the convenient sampling of which the participants were recruited. Findings might not be generalizable to children of relatively low socioeconomic status or those of mothers who do not have access to social media platforms.

\section{Conclusions}

The current study indicates that children exhibiting greater picky eating consume fewer vegetables and fruits, less protein, and higher amount of trans fat than children at the lower levels, presenting unhealthy dietary behaviors. The study could be replicated in more diverse populations to explore whether these observations are applicable to children of low socioeconomic status or food insecure families. Future studies may also investigate whether these dietary behaviors will remain into adulthood and examine the long-term effects of picky eating on cardiovascular health. Additionally, our findings highlight the need to explore how the obese mother-child relationship influences a child's picky eating behaviors. Dietary behaviors of mothers with obesity must be evaluated when designing intervention programs aiming to enhance dietary behaviors of children.

Funding: This research received no external funding.

Institutional Review Board Statement: The study was conducted according to the guidelines of the Declaration of Helsinki, and approved by Faculty of Applied Medical Sciences Ethics Committee of King Abdulaziz University (FAMS-EC-2020-0010).

Informed Consent Statement: Informed consent was obtained from all subjects involved in the study.

Data Availability Statement: The data used to support the findings of this study are available from the corresponding author upon request.

Acknowledgments: Thanks are due to all members of the Dietary Intake of Saudis project for their contribution to data collection. Special thanks to Fatima Abdulhakeem and Najwan Jannadi from the Nutrition Assessment Lab for their support during dietary data entry.

Conflicts of Interest: The author declares no conflict of interest.

\section{References}

1. Xue, Y.; Lee, E.; Ning, K.; Zheng, Y.; Ma, D.; Gao, H.; Yang, B.; Bai, Y.; Wang, P.; Zhang, Y. Prevalence of picky eating behaviour in Chinese school-age children and associations with anthropometric parameters and intelligence quotient. A cross-sectional study. Appetite 2015, 91, 248-255. [CrossRef]

2. Kutbi, H.A.; Alhatmi, A.A.; Alsulami, M.H.; Alghamdi, S.S.; Albagar, S.M.; Mumena, W.A.; Mosli, R.H. Food neophobia and pickiness among children and associations with socioenvironmental and cognitive factors. Appetite 2019, 142, 104373. [CrossRef]

3. Carruth, B.R.; Ziegler, P.J.; Gordon, A.; Barr, S.I. Prevalence of picky eaters among infants and toddlers and their caregivers' decisions about offering a new food. J. Am. Diet. Assoc. 2004, 104, 57-64. [CrossRef]

4. Jacobi, C.; Schmitz, G.; Agras, W.S. Is picky eating an eating disorder? Int. J. Eat. Disord. 2008, 41, 626-634. [CrossRef]

5. Mascola, A.J.; Bryson, S.W.; Agras, W.S. Picky eating during childhood: A longitudinal study to age 11 years. Eat Behav. 2010, 11, 253-257. [CrossRef] 
6. Goh, D.Y.; Jacob, A. Perception of picky eating among children in Singapore and its impact on caregivers: A questionnaire survey. Asia Pac. Fam. Med. 2012, 11, 1-8. [CrossRef]

7. Dovey, T.M.; Staples, P.A.; Gibson, E.L.; Halford, J.C. Food neophobia and 'picky/fussy'eating in children: A review. Appetite 2008, 50, 181-193. [CrossRef] [PubMed]

8. Taylor, C.M.; Northstone, K.; Wernimont, S.M.; Emmett, P.M. Macro-and micronutrient intakes in picky eaters: A cause for concern? Am. J. Clin. Nutr 2016, 104, 1647-1656. [CrossRef]

9. Jacobi, C.; Agras, W.S.; Bryson, S.; Hammer, L.D. Behavioral validation, precursors, and concomitants of picky eating in childhood. J. Am. Acad. Child. Adolesc. Psychiatry 2003, 42, 76-84. [CrossRef]

10. Tharner, A.; Jansen, P.W.; Kiefte-de Jong, J.C.; Moll, H.A.; van der Ende, J.; Jaddoe, V.W.; Hofman, A.; Tiemeier, H.; Franco, O.H. Toward an operative diagnosis of fussy/picky eating: A latent profile approach in a population-based cohort. Int. J. Behav. Nutr. Phys. Act. 2014, 11, 1-11. [CrossRef] [PubMed]

11. Cooke, L.; Wardle, J.; Gibson, E. Relationship between parental report of food neophobia and everyday food consumption in 2-6-year-old children. Appetite 2003, 41, 205-206. [CrossRef]

12. Van Der Horst, K.; Deming, D.M.; Lesniauskas, R.; Carr, B.T.; Reidy, K.C. Picky eating: Associations with child eating characteristics and food intake. Appetite 2016, 103, 286-293. [CrossRef] [PubMed]

13. Galloway, A.T.; Fiorito, L.; Lee, Y.; Birch, L.L. Parental pressure, dietary patterns, and weight status among girls who are "picky eaters". J. Am. Diet. Assoc. 2005, 105, 541-548. [CrossRef]

14. Taylor, C.M.; Wernimont, S.M.; Northstone, K.; Emmett, P.M. Picky/fussy eating in children: Review of definitions, assessment, prevalence and dietary intakes. Appetite 2015, 95, 349-359. [CrossRef]

15. Larsen, J.K.; Hermans, R.C.; Sleddens, E.F.; Engels, R.C.; Fisher, J.O.; Kremers, S.P. How parental dietary behavior and food parenting practices affect children's dietary behavior. Interacting sources of influence? Appetite 2015, 89, 246-257. [CrossRef]

16. Carruth, B.R.; Skinner, J.D. Revisiting the picky eater phenomenon: Neophobic behaviors of young children. J. Am. Coll. Nutr. 2000, 19, 771-780. [CrossRef] [PubMed]

17. Chao, H.-C. Association of picky eating with growth, nutritional status, development, physical activity, and health in preschool children. Front. Pediatrics 2018, 6, 22. [CrossRef] [PubMed]

18. Tharner, A.; Jansen, P.W.; Kiefte-de Jong, J.C.; Moll, H.A.; Hofman, A.; Jaddoe, V.W.; Tiemeier, H.; Franco, O.H. Bidirectional associations between fussy eating and functional constipation in preschool children. J. Pediatr. 2015, 166, 91-96.e91. [CrossRef] [PubMed]

19. Steinsbekk, S.; Bonneville-Roussy, A.; Fildes, A.; Llewellyn, C.H.; Wichstrom, L. Child and parent predictors of picky eating from preschool to school age. Int. J. Behav. Nutr. Phys. Act. 2017, 14, 87. [CrossRef]

20. Hulley, S.B.; Cummings, S.R.; Browner, W.S.; Grady, D.G.; Newman, T.B. Designing Clinical Research, 4th ed.; Gaertner, R., Ed.; Lippincott Williams \& Wilkins: Philadelphia, PA, USA, 2013.

21. Al-Qerem, W.A.; Ling, J.; AlBawab, A.Q. Validation of the comprehensive feeding practice questionnaire among school aged children in Jordan: A factor analysis study. Int. J. Behav. Nutr. Phys. Act. 2017, 14, 1-8. [CrossRef] [PubMed]

22. World Health Organization. Body Mass Index-BMI. Available online: https://www.euro.who.int/en/health-topics/diseaseprevention/nutrition/a-healthy-lifestyle/body-mass-index-bmi (accessed on 3 January 2021).

23. Wardle, J.; Guthrie, C.A.; Sanderson, S.; Rapoport, L. Development of the Children's Eating Behaviour Questionnaire. J. Child. Psychol. Psychiatry 2001, 42, 963-970. [CrossRef]

24. Alhamad, A.H. Validation of the Children's Eating Behaviour Questionnaire (CEBQ) in the Population of Saudi Preschoolers. Ph.D. Thesis, University of Waikato, Hamilton, New Zealand, 2013.

25. Finistrella, V.; Manco, M.; Ferrara, A.; Rustico, C.; Presaghi, F.; Morino, G. Cross-sectional exploration of maternal reports of food neophobia and pickiness in preschooler-mother dyads. J. Am. Coll. Nutr. 2012, 31, 152-159. [CrossRef]

26. Zuercher, J.L.; Wagstaff, D.A.; Kranz, S. Associations of food group and nutrient intake, diet quality, and meal sizes between adults and children in the same household: A cross-sectional analysis of US households. Nutr. J. 2011, 10, 1-12. [CrossRef] [PubMed]

27. Wroten, K.C.; O’Neil, C.E.; Stuff, J.E.; Liu, Y.; Nicklas, T.A. Resemblance of dietary intakes of snacks, sweets, fruit, and vegetables among mother-child dyads from low income families. Appetite 2012, 59, 316-323. [CrossRef] [PubMed]

28. McGowan, L.; Croker, H.; Wardle, J.; Cooke, L.J. Environmental and individual determinants of core and non-core food and drink intake in preschool-aged children in the United Kingdom. Eur J. Clin. Nutr 2012, 66, 322-328. [CrossRef]

29. Sonneville, K.R.; Rifas-Shiman, S.L.; Kleinman, K.P.; Gortmaker, S.L.; Gillman, M.W.; Taveras, E.M. Associations of obesogenic behaviors in mothers and obese children participating in a randomized trial. Obesity 2012, 20, 1449-1454. [CrossRef]

30. Taylor, C.M.; Steer, C.D.; Hays, N.P.; Emmett, P.M. Growth and body composition in children who are picky eaters: A longitudinal view. Eur. J. Clin. Nutr. 2019, 73, 869-878. [CrossRef]

31. Eicher-Miller, H.A.; Zhao, Y. Evidence for the age-specific relationship of food insecurity and key dietary outcomes among US children and adolescents. Nutr. Res. Rev. 2018, 31, 98-113. [CrossRef]

32. Mumena, W.A.; Kutbi, H.A. Household food security status, food purchasing, and nutritional health of Saudi girls aged 6-12 years. Prog. Nutr. 2021, 22. [CrossRef] 
33. Wang, Q.; Imamura, F.; Lemaitre, R.N.; Rimm, E.B.; Wang, M.; King, I.B.; Song, X.; Siscovick, D.; Mozaffarian, D. Plasma phospholipid trans-fatty acids levels, cardiovascular diseases, and total mortality: The Cardiovascular Health Study. J. Am. Heart Assoc. 2014, 3, e000914. [CrossRef] [PubMed]

34. Lichtenstein, A.H. Dietary trans fatty acids and cardiovascular disease risk: Past and present. Curr. Atheroscler. Rep. 2014, 16, 433. [CrossRef]

35. Emerging Risk Factors Consortium. Major lipids, apolipoproteins, and risk of vascular disease. JAMA 2009, 302, 1993-2000. [CrossRef]

36. Sacks, F.M.; Lichtenstein, A.H.; Wu, J.H.; Appel, L.J.; Creager, M.A.; Kris-Etherton, P.M.; Miller, M.; Rimm, E.B.; Rudel, L.L.; Robinson, J.G. Dietary fats and cardiovascular disease: A presidential advisory from the American Heart Association. Circulation 2017, 136, e1-e23. [CrossRef]

37. Kutbi, H.A. The Relationships between Maternal Feeding Practices and Food Neophobia and Picky Eating. Int J. Environ. Res. Public Health 2020, 17. [CrossRef]

38. Jansen, P.W.; de Barse, L.M.; Jaddoe, V.W.; Verhulst, F.C.; Franco, O.H.; Tiemeier, H. Bi-directional associations between child fussy eating and parents' pressure to eat: Who influences whom? Physiol. Behav. 2017, 176, 101-106. [CrossRef] 